\title{
The Korrontea Data Modeling
}

\author{
Emmanuel Bouix \\ LIUPPA - IUT de Bayonne \\ Château Neuf - Place Paul Bert \\ 64100 Bayonne, France \\ +33(0)559574326 \\ ebouix@acm.org
}

\author{
Philippe Roose \\ LIUPPA - IUT de Bayonne \\ Place Paul Bert \\ 64100 Bayonne, France \\ $+33(0) 559574348$ \\ roose@iutbayonne.univ-pau.fr
}

\author{
Marc Dalmau \\ LIUPPA - IUT de Bayonne \\ Château Neuf - Place Paul Bert \\ 64100 Bayonne, France \\ +33(0)559574321 \\ dalmau@ieee.org
}

\begin{abstract}
Needs of multimedia systems evolved due to the evolution of their architecture which is now distributed into heterogeneous contexts like the Internet network. A critical issue lies in the fact that they handle, process, and transmit multimedia data. This data integrates several properties which should be considered since it holds a considerable part of its semantic, e.g. the lips synchronization in a video. In this paper, we focus on the definition of a basic abstraction for describing and modeling data in multimedia systems by taking into account their properties. This abstraction is proposed with synchronization policies to ensure synchronous transport of multimedia data. We use it in a component model that we develop for the design and deployment of distributed multimedia systems.
\end{abstract}

\section{Categories and Subject Descriptors}

D.2.11 [Software Engineering]: Software Architectures - Data abstraction

\section{General Terms}

Algorithms, Management, Design.

\section{Keywords}

Distributed multimedia applications, data flows, temporal constraints, synchronization policies, component model.

\section{INTRODUCTION}

The aim of our works is to provide a global method for designing and developing distributed multimedia applications through the Internet. This work is QoS (Quality of Service) driven because these applications impose stringent requirements that the network layer of the Internet does not consider. Indeed, the quality required by end-users and the one provided by runtime environments are not taken into account. Thus, using these applications in such environments is compromised due to their moving and non-predictable characteristics (e.g. network bandwidth, terminal characteristics, operating system functionalities but also handicaps and languages of end-users). We define a software architecture suited to these applications [11]. They are composed of components connected together by connectors. These entities can be supervised by a middleware introduced to manage QoS [10], [12]. As an example of such applications, we can quote remote video monitoring which allows to monitor events or physical phenomena by using sensors like in [1]. We can use this kind of applications to keep watch on car parks or critical sections of roads where risks of traffic jam are higher [2]. Another more common example is videoconferencing

Permission to make digital or hard copies of all or part of this work for personal or classroom use is granted without fee provided that copies are not made or distributed for profit or commercial advantage and that copies bear this notice and the full citation on the first page. To copy otherwise, to republish, to post on servers or to redistribute to lists, requires prior specific permission and/or a fee. AMBI-SYS 2008, February 11-13, Quebec, Canada

Copyright @ 2008 ICST 978-963-9799-16-5

DOI 10.4108/ICST.AMBISYS2008.2896 systems which allow the meeting of several persons physically located in different places [3].

This paper introduces a data model that we use in this application architecture [11] in order to define a way for handling and transporting multimedia data. We call it "Korrontea" which means "the data flow" in the Basque language.

The rest of the paper is structured as follows: Section 2 provides some justification on the needs of such a model. Section 3 presents the Korrontea data model and its main characteristics. Section 4 describes briefly the Osagaia component model specified to develop these applications, the aim is to show the use of the Korrontea model. Section 5 presents the prototypes used to validate our works. Section 6 describes the related works. Section 7 provides some conclusions and discusses future work.

\section{WHY DO WE NEED A MODEL?}

We try to motivate our approach in detailing what we believe to be the important issues in modeling multimedia data.

\subsection{The Media}

The multimedia data handled by this kind of applications are generally called media. This term has a rich set of connotations. Media are form of information content where the goal is to inform or entertain end-users or audience. They are related to how information is conveyed and distributed. They exist in different forms and are very used in applications nowadays. Several research works are interested in the classification of multimedia applications [4], [5]. Some of them show the importance of data in such applications. In [5], the authors define multimedia applications as an information processing system which handles a combination of media like e.g. text, graphics, images, audio, video or control information.

Some media are constituted of a sequence of media elements, also called samples, which describe an information part represented by the media in an adequate coding format. They exist under the form of data flows. A data flow is a structure which provides information concerning the physical organization of samples, for instance their physical ordering and placement. Since these media are based on human sensory properties, their properties must be considered in applications. These media are called continuous. One of their particular characteristics is that they integrate synchronization relations between samples of both a single and several media [6]:

- intra-media synchronization refers to time relations between samples of the same media;

- inter-media synchronization refers to time relations between samples of several media.

These relations are very important for the media because they allow to achieve a natural impression at rendering time. Some studies on human perception of media and synchronization [7], 
[8] prove this viewpoint. Video and audio are examples of this kind of media.

We distinguish other kinds of media where the time factor is not preponderant. They are composed of a set of indivisible data necessary to render the media correctly. Habitually, they are called discrete media. For instance, an image is a discrete media composed of a finite set of pixels. Text and graphics are other examples.

Sometimes, it is necessary to synchronize discrete and continuous media in an inter-media way. This is the case of a video composed of audio, images and subtitles.

\subsection{Functional Specifications of Multimedia Applications}

In our works, the functional specifications of multimedia applications are described by means of graphs oriented and polar noted $\mathrm{G}\left(\mathrm{V}, \mathrm{E}_{\mathrm{s}}, \mathrm{E}_{\mathrm{c}}\right)$. We called them functional graphs; they are based on conditional process graphs described in [9]. More details about functional graphs are done in [10]. The set V represents the nodes of the graph where each of them represents a basic role or

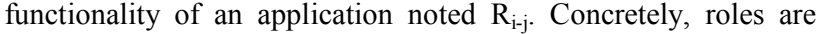
performed by either software or hardware components. $E_{s}$ and $E_{c}$ are the sets of simple and conditional edges. Whatever its type, an edge is used to link the output of a node to the input of another node. A conditional edge (set $\mathrm{E}_{\mathrm{c}}$ ) has an associated condition value. The paths described by these edges can be considered only if the associated condition value is true. They allow to indicate different configuration choices and thus constraints in the using of particular roles. A simple edge (set $E_{s}$ ) specifies that the node linked is an element of the application whatever the chosen configuration. Edges represent media, i.e. data of applications.

The functional graphs allow to specify inter-media synchronization by means of synchronization links between edges. Such links specify the fact that media must be kept synchronous during their transport in applications in spite of the processing that can be applied on them. Indeed, media processing introduces a problem that we identify as inter-media desynchronization. It is due to the fact that some media synchronized with others can be processed. Processing introduces temporal delays on media and so desynchronizes the processed media with the others.

Another source of inter-media desynchronization can occur when synchronous media are transmitted through the Internet network. Some services used to transport data on this network introduce an increase of the network load but also packet loss, delay and jitter [13]. These problems are well-known and harmful to media synchronization [14]

\subsection{Summary of Requirements}

The handling of media in applications is not an easy task. Indeed, this data has properties that we should consider in order to avoid the loss of their semantic. Thus, we propose to integrate media at design time by means of the Korrontea model that we present in this paper. In Table 1 , we summarize the requirements imposed by the handling of media and by the pattern given by the functional graphs.

We propose a data model which meets these requirements. The aim is to define a structure which allows integration of the media and in a general way of all the data that may exist in distributed multimedia applications: we propose to use data flows.
An advantage is to allow an easy integration of heterogeneous data.

Table 1. Requirements and Suggested Solutions

\begin{tabular}{|c|c|}
\hline Requirements/Specifications & Suggested Solutions \\
\hline $\begin{array}{c}\text { A structure which allows an easy integration of all the } \\
\text { kinds of media }\end{array}$ & Media are existing in the form of data flows \\
\hline $\begin{array}{l}\text { Considering the physical ordering of data that composes } \\
\qquad \text { a flow }\end{array}$ & Using an approach based upon a logical clock \\
\hline Considering the intra-media synchronization relations & Using an approach based upon a physical clock \\
\hline $\begin{array}{l}\text { Allowing the handling and processing of media in } \\
\text { applications }\end{array}$ & $\begin{array}{l}\text { Defining the internal structure of data flows handled by } \\
\text { implementation units }\end{array}$ \\
\hline $\begin{array}{l}\text { Allowing transmission of media through Intemet } \\
\text { network }\end{array}$ & Defining objects and mechanisms for this task \\
\hline Cassification of media & Based upon their temporal constraints \\
\hline Ensuring inter-media synchronization & Defining Synchronization policies \\
\hline
\end{tabular}

All the data has sequence and synchronization relations properties. The data which composes data flows is ordered with a sequence number given by a logical clock. Time stamps are used in order to define and consider the synchronization relations. We define synchronization policies with the aim of keeping these relations. These policies are based upon temporal behavior of data flows, i.e. the temporal constraints they integrate. Finally, this model proposes one way to handle, to process and to transfer media into applications in both local and distributed cases.

\section{THE KORRONTEA DATA MODEL}

Before giving details of the model, we give a representation of it under a formalism based on a UML [23] class diagram described in Figure 1.

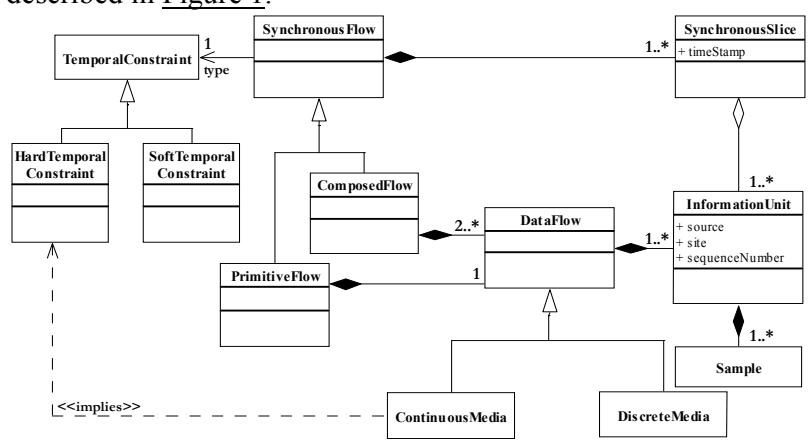

Figure 1. The Korrontea Data Flows Conceptual Model

\subsection{A Common Structure: the Data Flows}

The two kinds of media identified previously differ mainly by time constraints and data structure. This implies different ways for handling them and the necessity to know their types a priori. A lot of frameworks defined to provide multimedia programming proceed suchlike (see e.g. the sun one JMF [22]). Moreover, it is possible to define inter-media relations between data of different types. For facilitating this possibility and the handling of all kinds of media and data without a priori knowing their characteristics, we propose to use a unique abstraction: data flows. Such an abstraction is an interesting solution for the integration of media into these applications [5]. In functional graphs, we detail them by means of edges. Each edge takes its origin in one particular node of capture or creation. Thus, a data flow is produced by a unique 
concrete component located on a unique site. These particular components are called located sources.

\section{Definition 1 Located Sources}

We call a located source $L S$ a couple $(S, L)$ where $S$ is a component and $L$ the site where $S$ is located.

An important notion brought by this component is the characteristic of distribution. An important property of these components is the site where they are located.

Data flows are composed of a sequence of data called samples. They include such things as video-frames, images, text, audio samples, events, etc.

Definition 2 Data Flows

$A$ data flow $f$ is composed of a possibly infinite sequence of samples with finite size. Each sample of $f$ is produced by the same located source $L S=(S, L)$. Attributes of a data flow:

- $\quad$ locatedSource $(f)=L S$

- $\quad \operatorname{source}(f)=S$

- $\quad$ site $(f)=L$ (capture or creation site of the flow)

The creation of data flows by located sources consists in the continuous production of samples at either regular or irregular rate. Samples are produced in an adequate coding format which describes the information transported by the flow. We consider that all data flows are a priori composed of an infinite sequence of samples. Undoubtedly, data flows composed of a finite sequence can exist too, as in the case of data flows stored in files.

When data flows have no more samples that mean that this data is not used any more by an application. In such a case, these flows and components which produced and processed them must be removed from the application. It is important to notice that the operators, properties and policies that we define can be only applied onto data flows which have samples.

At creation time but also during the handling and processing of samples, the property of sequence must be explicitly defined. For that reason, we use a logical clock to stamp each sample or set of samples with an integer which allows to distinguish two successive samples or set of samples and so allows to maintain this property. Each component of an application provides, for each flow it produces, a mechanism which produces incremented integers. These integers are called sequence numbers. This concept was initially defined by Lamport [15] and classically used in distributed systems to preserve the order of data production [16], [17].

Handling data flows in applications depends on intrinsic characteristics of the supported information. A media can be seen according to several hierarchical levels where each of them describes a different granularity. For instance, a video can be decomposed into scenes, images, blocks and pixels. Applications are defined to handle one of these units. However, when a finegrained one is chosen, like pixel for a video, it will be difficult or even impossible to keep the intra-media relations in a software way. Some works [18] try to provide hardware solutions for the handling and processing of fine-grained units of media. In order to avoid this problem, it is necessary to consider a sufficient number of samples. We propose to gather sufficient set of samples into information units. For instance, audio data are handled into programs by means of audio segment which gather samples which correspond to approximately 200 milliseconds [25]. This quantity of information is associated with the sequence number. In [5], the authors use nearly the same idea through the concept of Logical Data Units (LDU).
Definition 3 Information Units

We call an information unit $I U$, a couple $(E, i)$ where $E=\left\{e_{1}, e_{2}\right.$, $\left.\ldots, e_{n}\right\}$ is a finite set of samples of the same data flow $f$ and $i$ an integer used to define the sequence property for this data flow. We will see that this property is defined between information units of the same data flow. Attributes of information units:

- $\quad$ flow $(I U)=f$ (data flow from whom elements of $E$ belongs to)

- $\quad \operatorname{samples}(I U)=E$

- $\quad$ sequenceNumber $(I U)=i$

- $\quad$ locatedSource $(I U)=$ locatedSource(flow(IU))

- $\operatorname{source}(I U)=\operatorname{source}($ flow $(I U))$

- $\quad$ site $(I U)=\operatorname{site}(f l o w(I U)$ ) (capture or creation site of the unit)

The constitution of information units depends on the considered media and on the specifications of the application. For example, in a video we can gather the pixels which constitute an image. According to the specifications, we can decide to compose information units by one, two or three images, etc.

Therefore, data flows are composed of sequence of information units. Two successive units are separated by certain durations. The creation of a unit is done at a particular time earlier than the creation of the next one. Physical clocks are mechanisms used to define temporal values and to stamp data in distributed systems. The main difference with logical one is that with a physical clock, we manipulate real time. Thus, we can handle time value, time interval and duration. More details about physical clocks can be found in [19] where a time model is defined. Physical clocks can be global or local. Global ones allow to dispose of a unique temporal reference in distributed systems. A time value provided by these clocks describes the same physical instant on every site where such a system is deployed. Several research works deal with this solution. Their definition is based upon clocks at each site. These approaches impose approximations due to temporal skews of clocks in relation with others [20]. This kind of research is based upon both probability and statistical approaches. Such a solution will probably influence the degree of synchronization because it is impossible to have a perfect and absolute synchronization of clocks through the Internet network [21]. Consequently, it is difficult to use a global clock without introducing a margin of error. A second approach consists in using local physical clocks. This solution recommends the use of physical clocks at each site where a distributed system is deployed. The time can be handled but in this case relatively to the clock of a particular site: temporal relations can be defined and kept between data created on the same site. We consider that the synchronization of media created or captured on different sites is something artificial and so it is not necessary to have strict temporal relations for this task. Our purpose is not to create synchronization but to keep it in order to prevent the loss of information (the one of synchronization) which existed at creation time of several media coming from the same site. On the basis of these arguments, we choose to use an approach based upon local physical clocks. Thus, located sources presented in definition 1 allow to introduce this notion of localization. These particular components take their meaning in the following definitions and properties.

Definition 4 Local Physical Clock

We assume that in every site $L$, there is a unique component $C_{p}$ (local physical clock of $L$ ). Samples produced by $L S=\left(C_{p}, L\right)$ are integers strictly increasing called time stamps. 
These integers considered independently of each other have no meanings. Nevertheless, they are linked to real time by a proportionality ratio. Thus, if LS produces integer $n_{1}$ at the time value $t_{1}$ and integer $n_{2}$ at the time value $t_{2}$, then $\left(n_{2}-n_{1}\right)=k_{L}\left(t_{2}-t_{1}\right)$ where $\mathrm{k}_{\mathrm{L}}$ is a constant known in site $\mathrm{L}$.

Assumption 1 Time Constant

On each site of a multimedia application, constant $k_{L}$ is the same and is equal to $k$.

This assumption means that all the local physical clocks of an application have the same rate. Time values produced by local physical clocks $\left(\mathrm{C}_{\mathrm{p} 1}, \mathrm{~L}_{1}\right)$ and $\left(\mathrm{C}_{\mathrm{p} 2}, \mathrm{~L}_{2}\right)$ cannot be compared; we can only compare differences between time values produced respectively by these located sources. This is an important characteristic of our model. Indeed, knowing the constant $\mathrm{k}_{\mathrm{L}}$ allows to respect intra-flow synchronization relations at rendering time and whatever the site where data flows come from.

Synchronization between data flows which come from the same site can be kept during their transport with the time stamps done by local physical clocks. We define an object that we call synchronous slice. It is used to gather a set of information units created or captured in the same site and which corresponds to the same time interval.

\section{Definition 5 Synchronous Slices}

Let $F=\left\{f_{1}, f_{2}, \ldots, f_{n}\right\}$ a set of data flows such as $\forall f_{i} \in F$, site $\left(f_{i}\right)=L$. We call a synchronous slice, defined on $F$, an object which contains an information quantity which corresponds to the same time interval and that we define as follows: $S S=(t, U)$ where $t$ is a time stamp created by $L S=\left(C_{p}, L\right)$ and $U$ is a finite set of information units such as $\forall x \in U$, flow $(x) \in F$. Sequence numbers of information units are defined in order to respect the following property: let $A_{f}=\{e \in U /$ flow $(e)=f\}$ then $\forall e \in A_{f}, 1 \leq$ sequenceNumber(e) $\leq\left|A_{f}\right|$ (cardinal of set $A_{f}$ ) and $\forall e_{1}, \forall e_{2} \in A_{f}$, $e_{1} \neq e_{2} \Leftrightarrow$ sequenceNumber $\left(e_{1}\right) \neq$ sequenceNumber $\left(e_{2}\right)$.

Attributes of Synchronous Slices:

- $f l o w(S S)=F$ (this attribute allows to know the data flows of a particular synchronous slice)

- $\quad$ timeStamp $(S S)=t$

- $\quad$ informationUnits $(S S)=U$

- $\quad \operatorname{site}(S S)=L$

The time stamps are assigned to slices after the creation of information units that will be gathered in them. In a same slice, the information units are stamped with sequence numbers.

Synchronous slices are the units of synchronous transport of data in applications. We define synchronous flows that are composed of a sequence of synchronous slices.

Definition 6 Synchronous Flows

A synchronous flow $S F$ is composed of a possibly infinite sequence of synchronous slices with the same flow set such as $\forall$ $S S \in S F$, flow $(S S)=F$ (see definition 5). Synchronous slices of a same synchronous flow have different time stamps, i.e.: $\forall S S_{1}, \forall$ $S S_{2} \in S F, S_{1} \neq S S_{2} \Leftrightarrow \operatorname{timeStamp}\left(S S_{1}\right) \neq \operatorname{timeStamp}\left(S S_{2}\right)$.

Property of Synchronous Flows:

- $\forall S S_{1}, \forall S S_{2} \in S F$, site $\left(S S_{1}\right)=\operatorname{site}\left(S S_{2}\right)=L$

- Proof: According to this definition, we have $\forall S S_{1}, \forall S S_{2} \in$ $S F$ : flow $\left(S S_{1}\right)=$ flow $\left(S S_{2}\right)=F$. According to definition 5, $F$ is a set of data flows such as $\forall f_{i} \in F$, site $\left(f_{i}\right)=L$

Attributes of Synchronous Flows:

- $f l o w(S F)=F$ where $F$ is the set of data flows (see definition 2) which composes synchronous flows
- $\quad$ site $(S F)=L$ (all the flows, which compose synchronous slices of $S F$, come from the same site)

An information unit is an element of one and only one synchronous slice. In the same way, a synchronous slice is an element of one and only one synchronous flow.

Property 1 Property of Information Units Membership

- synchronousSlice $(I U)=S S \in S F$ such as $I U \in$ informationUnits(SS)

According to the composition of synchronous slices, we distinguish two kinds of synchronous flows: the primitive one and the composed one.

\section{Definition 7 Primitive Synchronous Flows}

A synchronous flow is defined as primitive when $\mid$ flow $(S S) \mid=1$.

Data flows linked by inter-flow synchronization relations are put in composed flows. For each data flow, synchronous slices of composed flows contain information units which correspond to the same time interval. The provided policies that we will describe are used to create composed flows.

\section{Definition 8 Composed Synchronous Flows}

A synchronous flow is called composed when $\mid$ flow $(S S) \mid>1$.

Information units of synchronous flows can be ordered by time and by an integer which represents the sequence. We define strict total order relations between information units of a same synchronous flow. This property can be used at presentation time of data in order to have ordered rendering.

Property 2 Strict Total Order Relations $(<$ and $>$ ) between information units of a data flow into a synchronous flow $S F$

We define $<$ (respectively $>$ ) as a strict total order relation between information units of a same synchronous flow. Let $I U_{1}$ and $I U_{2}$ two information units $\in S F$, with flow $\left(I U_{1}\right)=$ flow $\left(I U_{2}\right)$. We have:

- $t_{1}=$ timeStamp(synchronousSlice $\left.\left(I U_{1}\right)\right)$,

$n_{1}=$ sequenceNumber $\left(I U_{1}\right)$

- $t_{2}=$ timeStamp $\left(\right.$ synchronousSlice $\left.\left(I U_{2}\right)\right)$

$n_{2}=$ sequenceNumber $\left(I U_{2}\right)$

$I U_{1}<I U_{2}$ (respectively $\left.I U_{1}>I U_{2}\right) \Leftrightarrow\left(t_{1}<t_{2}\right)$ OR $\left(\left(t_{1}=t_{2}\right) \quad A N D\right.$ $\left.\left(n_{1}<n_{2}\right)\right)$ (respectively $\left(t_{1}>t_{2}\right)$ OR $\left.\left(\left(t_{1}=t_{2}\right) A N D\left(n_{1}>n_{2}\right)\right)\right)$. If $t_{1} \neq t_{2}$, the order is done by the time stamp. If $t_{1}=t_{2}$, the order is done by the sequence number.

Proof: A strict order has properties of irreflexivity and transitivity. Moreover, this order is total if we have: $\forall I U_{1}$ and $I U_{2}$ two information units $\in S F$, with flow $\left(I U_{1}\right)=$ flow $\left(I U_{2}\right)$, $I U_{1}<I U_{2}$ OR IU $U_{1}=I U_{2}$ OR IU $U_{2}<I U_{1}$.

- Irreflexivity: $\forall x \in S F$, let SS a synchronous slice / $x \in$ informationUnits(SS).

timeStamp(synchronousSlice $(x)$ ) $<$ timeStamp(synchronousSli $c e(x))$ is false because these two time stamps are equals. In the same way, sequenceNumber $(x)<$ sequenceNumber $(x)$ is false because these two numbers are equals. Hence, relation $x<x$ is false.

- Transitivity: $\forall I U_{1}, I U_{2}$ and $I U_{3}$ three information units $\epsilon$ $S F$, with flow $\left(I U_{1}\right)=$ flow $\left(I U_{2}\right)=$ flow $\left(I U_{3}\right)$ such as $I U_{1}<I U_{2}$ and $I U_{2}<I U_{3}$. We have: $t_{1}=$ timeStamp $\left(\right.$ synchronousSlice $\left.\left(I U_{1}\right)\right)$,

$n_{1}=$ sequenceNumber $\left(I U_{1}\right)$ and

$t_{2}=$ timeStamp $\left(\right.$ synchronousSlice $\left.\left(I U_{2}\right)\right)$.

$n_{2}=$ sequenceNumber $\left(I U_{2}\right)$

and

$t_{3}=$ timeStamp (synchronousSlice $\left(I U_{3}\right)$ ),

$n_{3}=$ sequenceNumber $\left(I U_{3}\right)$. 
- if I $U_{1}<I U_{2}$ is due to the fact that $t_{1}<t_{2}, I U_{2}<I U_{3} \Rightarrow t_{2} \leq t_{3}$, so we have $t_{1}<t_{2} \leq t_{3} \Rightarrow I U_{1}<I U_{3}$.

- if $I U_{1}<I U_{2}$ is due to the fact that $t_{1}=t_{2} A N D n_{1}<n_{2}$ and $I U_{2}<I U_{3}$ is due to the fact that $t_{2}<t_{3}$, so we have $t_{1}=t_{2}<t_{3} \Rightarrow$ $I U_{1}<I U_{3}$. If $I U_{2}<I U_{3}$ is due to the fact that $t_{2}=t_{3} A N D n_{2}<n_{3}$, so we have $t_{1}=t_{2}=t_{3}$ and $n_{1}<n_{2}<n_{3} \Rightarrow I U_{1}<I U_{3}$

Hence, $<$ is a strict order relation. The same proof can be established for relation $>$.

Now, we must prove that these relations are total. Let $t_{1}=$ timeStamp $\left(\right.$ synchronousSlice $\left.\left(I U_{1}\right)\right), n_{1}=$ sequenceNumber $\left(I U_{1}\right)$ and $t_{2}=$ timeStamp(synchronousSlice $\left(I U_{2}\right)$ ), $n_{2}=$ sequenceNumber $\left(I U_{2}\right), t_{1}$ and $t_{2}$ are produced by a physical clock, they are integers and we know that $<$ is a total order relation on integers, so we have:

- $t_{1}<t_{2} \Rightarrow I U_{1}<I U_{2}$

- $\quad$ or $t_{2}<t_{1} \Rightarrow I U_{2}<I U_{1}$

- or $t_{1}=t_{2}: I U_{1}$ and $I U_{2}$ are information units which verify flow $\left(I U_{1}\right)=$ flow $\left(I U_{2}\right)=f$. According to the definition 5, we know that $\forall e_{1}, \quad \forall \quad e_{2} \in A_{f}, e_{1} \neq e_{2} \Leftrightarrow$ sequenceNumber $\left(e_{1}\right) \neq$ sequenceNumber $\left(e_{2}\right)$, so we know that $I U_{1} \neq I U_{2} \Leftrightarrow n_{1} \neq n_{2}$ and by contraposition $I U_{1}=I U_{2} \Leftrightarrow n_{1}=n_{2}$. The relation $<$ is a total order on integers, so we have:

$$
\begin{aligned}
& \text { - } n_{1}<n_{2} \Rightarrow I U_{1}<I U_{2} \\
& \text { - or } n_{2}<n_{1} \Rightarrow I U_{2}<I U_{1} \\
& \text { - or } n_{2}=n_{1} \Rightarrow I U_{2}=I U_{1}
\end{aligned}
$$

Thus, $<$ (respectively $>$ ) is a strict total order relation.

The relation $<$ means "earlier than" and the relation $>$ means "later than". In the same way, we define strict total order relations between synchronous slices of synchronous flows.

Property 3 Strict Total Order Relations (< and $>$ ) between synchronous slices of a synchronous flow $S F$

We define $<$ (respectively $>$ ) as a strict total order relation between synchronous slices of a same synchronous flow by: $\forall S S_{1}$ $\in S F, \quad \forall S S_{2} \in S F, \quad S S_{1}<S S_{2}$ (respectively $\quad S S_{1}>S S_{2}$ ) $\Leftrightarrow$ timeStamp $\left(S S_{1}\right)<\operatorname{timeStamp}\left(S S_{2}\right)\left(\right.$ respectively timeStamp $\left(S S_{1}\right)>$ timeStamp $\left.\left(\mathrm{SS}_{2}\right)\right)$.

Proof: $\forall S S_{1}, \quad S S_{2} \in S F$ with $S S_{1} \neq S S_{2}$, we have timeStamp $\left(S S_{1}\right) \neq$ timeStamp $\left(S S_{2}\right)$ (see definition 6). Moreover, timeStamp() is an integer and so we have timeStamp $\left(S S_{1}\right)<$ timeStamp $\left(S S_{2}\right)$ or timeStamp $\left(S S_{1}\right)>\operatorname{timeStamp}\left(S S_{2}\right)$. We have also $S S_{1}<S S_{2}$ or $S S_{1}>S S_{2}$. Thus, all synchronous slices of a synchronous flow can be ordered by relations $<$ and $>\Rightarrow$ these relations are strict total order between synchronous slices of a synchronous flow.

Property 4 Properties of synchronous flows (part 2)

Synchronous slices of a synchronous flow are totally ordered by $<$ and $>$ strict total order relations (see property 3). Information units of slices are totally ordered by $<$ and $>$ strict total order relations (see property 2).

Thanks to these relations, we can handle sequence and time. We define operators which permit, for each synchronous slice, to handle the sequence in flows. For each slice we can know the previous and the next ones.

Definition 9 Previous (prev) and Next (next) Operators

According to the property 3, synchronous slices of a synchronous flow SF are totally ordered by $<$ and $>$ relations. So, we can define:

- $\quad \forall S S_{1} \in S F, \exists S S_{2} \in S F$ called prev $\left(S S_{1}\right) / S S_{2}<S S_{1}$ and $\forall$ $S S_{3} \in S F$, we have: $S S_{3}<S S_{2}$ or $S S_{3}>S S_{1}$
- $\quad \forall S S_{1} \in S F, \exists S S_{4} \in S F$ called next $\left(S S_{1}\right) / S S_{4}>S S_{1}$ and $\forall$ $S S_{3} \in S F$, we have: $S S_{3}>S S_{4}$ or $S S_{3}<S S_{1}$

We can handle time intervals too. The using of a local physical clock imposes that these intervals are defined between slices with the same site of capture/creation.

Definition 10 Time Intervals

We define time intervals between synchronous slices of a synchronous flow $S F$ as follows: $\forall S S_{1}, S S_{2} \in S F$, we define $<S S_{1}, S S_{2}>=\mid \operatorname{timeStamp}\left(S S_{2}\right)$-timeStamp $\left(S S_{1}\right) \mid$.

\subsection{The Temporal Constraints}

In this model, the data must be classified according to their temporal behaviors in order to handle its in an efficient way in applications. Such a classification depends on the specifications of applications to develop. We mean it depends on the media used. The relevance of this classification is guided by the fact that the temporal behavior defines the way of handling media. Thus, we distinguish synchronous flows with either soft or hard temporal constraints. We consider that continuous media have hard temporal constraint due to their types of information and to their types of temporal constraints (studies on human perception of media argue this viewpoint [7], [8]) and also because nonobservance of these constraints involves the loss of the media semantics. In the UML diagram, we specified this property by means of an $<<$ implies $>>$ dependency [23] (see Figure 1). On the other hand, discrete media can be with hard or soft temporal constraints.

\section{Property 5 Hard Temporal Constraint}

A synchronous flow has hard temporal constraints if, $\theta$ being defined for an application, $\forall S S \in S F /<S S$, next(SS) $>\leq \theta$.

For distinguishing the temporal constraints of data, we introduce a parameter $\theta$ which must be defined at design time. It represents the maximal time value between two successive synchronous slices.

Property 6 Soft Temporal Constraint

A synchronous flow has soft temporal constraints if, $\theta$ being defined for an application, $\exists S S \in S F /<S S$, next $(S S)>>\theta$.

The synchronization policies that we will define in the next section are based upon this. Moreover, components of an application will know how to handle data thanks to these constraints.

\subsection{Multimedia Synchronization Policies}

We saw that it is essential to ensure synchronization. Indeed, without synchronization the media look somewhat artificial and incomprehensible [7], [8]. Functional graphs allow specifying inter-flow relations. This specification means that flows linked by this way must be kept synchronous during their transport into applications. Intra-flow relations are not clarified because they are explicitly defined in synchronous flows by means of time stamps.

\subsubsection{Intra-Flow Synchronization}

These relations correspond to the rate of flows; they give temporal relations between data which compose a flow. For instance, a 25 image per second rate video needs displaying one image every 40 milliseconds. These relations are not strict, tolerances may be accepted [7], [8]. The rendering of synchronous flows must be defined at design time and implemented in rendering components. For each synchronous flow, we can define intra-flow synchronization by using the sequence number of information units and the time stamp of synchronous slices. The 
strict total order relations permit to order all information units of these flows and so all samples. Temporal constraints of these flows determine the kind of intra-flow synchronization in relation with the time value $\theta$ defined at design time.

Sequence numbers, time stamps and temporal constraints are defined for each flow at creation or capture time by located sources (see definition 1). Some processing and handling of flows can affect this information. In order to avoid this problem, we are planning to update, by particular services attached to the components, these characteristics during the transport of flows into applications. These services are described by the component model defined for the implementation of these applications [12].

\subsubsection{Inter-Flow Synchronization}

Inter-flows synchronization corresponds to temporal relations that may exist between data of several flows. This is for instance the relations that link audio and images in a video. They are described in functional graphs by mean of synchronization links. In our works, this kind of synchronization can be ensured between flows captured or created in the same site.

Keeping these relations requires expressing relations between synchronous slices of different flows. We define these relations by providing operators that return particular time stamps used by policies in order to handle synchronous slices.

Definition 11 minimalTimeStamp and maximalTimeStamp Operators

We define operators that return the synchronous slices with the smallest and the greatest time stamp from a set of slices $E . E=\left\{e_{1}\right.$, $\left.e_{2}, \ldots, e_{n}\right\}$ with $\forall e_{i}, \forall e_{j} \in E$, site $\left(e_{i}\right)=\operatorname{site}\left(e_{j}\right)$, such as:

- $\quad \operatorname{minimalTimeStamp}(E)=\min _{\left.e_{i} \in E^{(\operatorname{timeStamp}}\left(e_{i}\right)\right)}$

- $\quad \operatorname{maximalTimeStamp}(E)=\max _{\left.e_{i} \in E^{(\operatorname{timeStamp}}\left(e_{i}\right)\right)}$

Several synchronous slices issue from the same site can have the same time stamp. Consequently, more than one element of a set $\mathrm{E}$ can match to minimalTimeStamp(E) (respectively maximalTimeStamp(E)).

Now, we define operators that may be applied on a set of slices coming from one or several synchronous flows.

Definition 12 First Occurrence Operator on a set of Synchronous Flows

Let $S G=\left\{f_{1}, f_{2}, \ldots, f_{n}\right\}$ a set of synchronous flows such as $\forall f_{i} \in$ $S G$, site $\left(f_{i}\right)=L$. On such a set, we define an operator called firstOccurence ${ }_{S G}(t)$ as follows:

- firstOccurence $_{S G}(t)=$ minimalTimeStamp $(E)$ with $E=\left\{e_{i} \in f\right.$ / timeStamp $\left(e_{i}\right) \geq t$ with $\left.f \in S G\right\}$

Definition 13 Last Occurrence Operator on a set of Synchronous Flows

Let $S G=\left\{f_{1}, f_{2}, \ldots, f_{n}\right\}$ a set of synchronous flows such as $\forall f_{i} \in$ $S G$, site $\left(f_{i}\right)=L$. On such a set, we define an operator called lastOccurence $_{S G}(t)$ as follows:

- $\quad \forall f \in S G$, we define the set firstSlice $f(t)$ by: firstSlice $_{f}(t)=\{S S$ / SS $\in f$ such as timeStamp(SS) $\geq t$ and timestamp(prev(SS))

$<t\}$. This set contains first synchronous slice of $f$ which time stamp is greater or equal to $t$.

- $\quad$ lastOccurence $_{S G}(t)=$ maximalTimeStamp $\left(E^{\prime}\right)$ with

$$
E^{\prime}=\bigcup_{i} \in S G \text { firstislice } f_{i}{ }^{(t)}
$$

Property 7 Properties of Occurrence Operators

- lastOccurence $_{S G}(t) \geq$ firstOccurence $_{S G}(t) \geq t$
- Proof: $\exists e \in f$ such as: $\operatorname{timeStamp}(e)=$ $\operatorname{minimalTimeStamp}(E)=\min _{e_{i} \in E^{(}}\left(\operatorname{timeStamp}\left(e_{i}\right)\right) W e$

will demonstrate that $e \in$ first $_{\text {Slice }}(t)$. We know that timeStamp(e) $\geq t$ (see definition 13). prev(e) verifies property timeStamp(prev(e)) $<$ t because if not that means that prev(e) $\epsilon E$ and in this case we would have minimalTimeStamp $(E)=\operatorname{timestamp}(\operatorname{prev}(e))<\operatorname{timeStamp}(e)$ because prev(e)<e (see definition 9). Hence, we have $e \in$ firstSlice $_{f}(t)$, lastOccurrence $_{S G}(t)=$ maxsimalTimeStamp $\left(E^{\prime}\right) \quad$ with $E^{\prime}=\bigcup_{f_{i} \in S G}$ firstSlice $_{f_{i}}(t)$, so $e \in E^{\prime}$ and maximalTimeStamp (E') \timeStamp(e). Hence, we have lastOccurence $_{S G}(t)$ firstOccurrence $_{S G}(t)$. Moreover, firstOccurrence $_{S G}(t)=$ minimalTimeStamp $\left(\left\{e_{i} \in f /\right.\right.$ timeStamp $\left.\left.\left(e_{i}\right) \geq t\right\}\right) \geq t$ because it is defined such as synchronous slices which have the minimum time stamp greater or equal to $t$.

The aim of these policies is to define composed flows and so their synchronous slices. Composition depends on the temporal constraints of the synchronous flows used. Policies are applied on set of synchronous flows. Such a set can contain indifferently primitive or composed flows. The policies produce composed flows.

Property 8 Property of a set of Synchronous Flows

- A set of Synchronous Flows can be decomposed into two subsets as follows: $S G=\left\{S G_{\text {hard }}\right\}\left\{\left\{S G_{\text {soff }}\right\}\right.$. Thus, $S G_{\text {hard }}=\left\{f_{i}\right.$ $\in S G / f_{i}$ is a flow with hard temporal constraint $\}$ and $S G_{\text {soft }}=\left\{f_{i} \in S G / f_{i}\right.$ is a flow with soft temporal constraint $\}$.

This property is important because it allows to define the way to constitute the synchronous slices of the composed flows. According to the constraints of synchronous flows, we define three different policies:

- the first one is applied when the set of synchronous flows contains only flows with hard temporal constraint;

- the second one is applied when the set of synchronous flows contains flows with both constraints;

- the third one is applied when the set of synchronous flows contains only flows with soft temporal constraint.

In these policies, we consider the fact that synchronous flows can arrive ahead of time or after the ones in relation with the others. We take into account these possible temporal delays between flows and so propose efficient policies. To do this, we introduce a parameter $\alpha$ which represents the maximum delay of synchronous slices on each flow. After, this delay we consider that all the slices of all the flows are available.

\subsubsection{Hard Policy: $S G_{\text {hard }} \neq \varnothing$ and $S G_{\text {soft }}=\varnothing$}

The principle of hard policy is to make synchronous slices of the resulting composed flow by gathering every slice of whose time stamp is included between the first occurrence and the last occurrence. This guarantees that every resulting slice will contain at least one information unit of each flow. In synchronous flows with hard temporal constraint, we are sure to receive a slice with a maximal delay of $\alpha+\theta$.

The hard policy algorithm is done in Figure 2. The first task consists in waiting at least one synchronous flow on each flow. On the set formed by these slices, we apply the operator lastOccurence in order to determine a time value called $\mathrm{T}_{\text {MAX }}$. In 
order to compensate time delays between flows, we wait for the slices which verify timeStamp(SS) $>\mathrm{T}_{\mathrm{MAX}}$. Once these tasks have ended, we can constitute the resulting slice by adding every information unit of the received slices which verify timeStamp(SS) $\leq \mathrm{T}_{\mathrm{MAX}}$. Then, we assign successive sequence number to each information unit. The time stamp of the resulting slice will be equal to firstOccurence applied to the slices used to compose it.

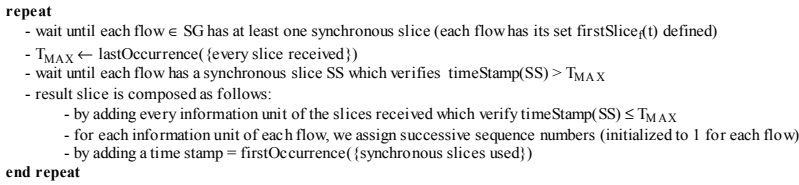

Figure 2. Hard Policy Algorithm

\subsubsection{Mixed Policy: $S G_{\text {hard }} \neq \varnothing$ and $S G_{\text {sofi }} \neq \varnothing$}

In mixed policy, we compose resulting slices by gathering every slice of synchronous flows where the time stamp is included between firstOccurrence and lastOccurrence applied in the subset SGhard. This guarantees that the resulting slice contains at least one information unit on each flow element of SGhard. Nevertheless, flows element of SGsoft can have information units or not. We use a delay equal to $\alpha$ in order to wait for synchronous slices of flows with soft temporal constraint, beyond which we consider that all the slices of these flows have been received.

This algorithm is more complex than the previous because it is necessary to consider the flows with soft temporal constraint where it is not possible to know a priori if data is available or not. Thus, we propose to use a principle similar to the one of semaphore in operating system field. Each arrival of a slice on one of the flow with hard temporal constraint triggers a delay $\alpha$. At the end of this delay, an authorization of slice constitution is done. This mechanism allows to take into consideration the possible delay of receiving slices of same time stamp in other flows. Every slices received during this delay will not be obligatorily put in the same slice than the one which triggered the delay. Indeed, they will put in the same slice only if their time stamps verify the adequate properties.

The algorithm of this policy is done onto Figure 3. First, we initialize the variable "autorisation" to 0 . Then, we wait on each flow with hard temporal constraint at least one synchronous slice. When a slice is available on these synchronous flows, we run a timer with a delay $\alpha$. At each end of the delay, the process executes: autorisation $\leftarrow$ autorisation +1 . $\mathrm{T}_{\mathrm{MAX}}$ is defined with the operator lastOccurence applied on every synchronous slices received. We wait until each flow with a hard temporal constraint has a synchronous slice $\mathrm{SS}$ which verifies timeStamp(SS) $>\mathrm{T}_{\mathrm{MAX}}$. Finally, the resulting slice is composed by adding every information unit of slices received whose time stamps verify timeStamp(SS) $\leq \mathrm{T}_{\mathrm{MAX}}$. Each of these units is associated with a sequence number initialized to 1 for each flow. The time stamp of the resulting slice will be equal to firstOccurrence applied on synchronous slices of flows with hard temporal constraint used. The variable "autorisation" is decreased by the number of slices of flows with hard temporal constraint used.

\subsubsection{Soft Policy: $S G_{\text {hard }}=\varnothing$ and $S G_{\text {sofi }} \neq \varnothing$}

The last policy consists in composing the resulting slices by gathering every received slice whose time stamp is included between firstOccurrence and firstOccurrence $+\theta$. This guarantees that a slice will contain at least one information unit. We wait for the first synchronous slice which allows defining firstOccurrence.

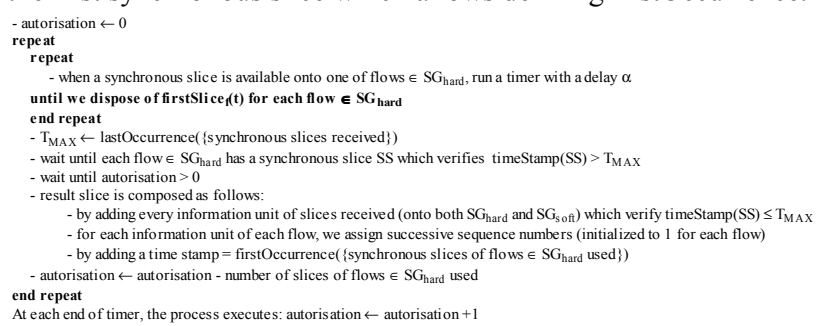

Figure 3. Mixed Policy Algorithm

We put in the resulting slices every slice whose time stamp does not exceed firstOccurence of a delay $\theta$. Using $\theta$, which constitutes the limit between both hard and soft temporal constraints, implies that we consider synchronous slices produced in this time interval. In this policy, we use the same mechanism than in the previous one. The authorization of the slices constitution is now based on a delay $\alpha+\theta$.

The soft policy algorithm is detailed in Figure 4. We initialize the "autorisation" variable to 0 . As soon as a slice is available on one of the synchronous flows with soft temporal constraint, a timer is run with a delay $\alpha+\theta$. When autorisation is strictly greater than 0 , we can begin to compose the resulting slice by adding every slice received which verifies timeStamp(SS) $\leq$ firstOccurence $(\{$ slices received $\})+\theta$. Information units are associated with a sequence number. The time stamp of the resulting slice is equal to firstOccurrence applied on slices used to compose it. Finally, the variable "autorisation" is decreased by the number of slices used.

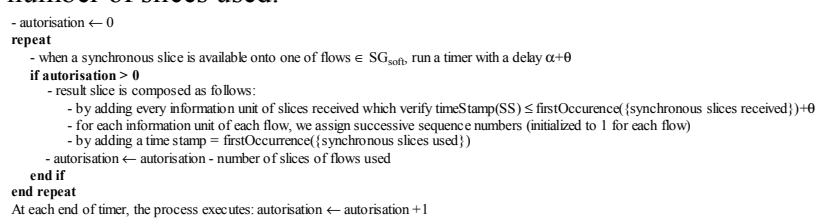

Figure 4. Soft Policy Algorithm

\subsubsection{Notice}

The mechanism used to manage temporal delays is based upon the semaphore mechanism. At each end of the delay, we give an authorization to make up a slice by executing autorisation $\leftarrow$ autorisation +1 . In the same way, each composition of the resulting slices corresponds to the consumption of such authorizations.

The delay $\alpha$ introduced to compensate delay between flows allows to synchronize these flows by considering margin of errors. If we choose for $\alpha$ a low value, we do not wait all the slices on all the flows and so the temporal relations between flows will introduce delays. If we choose for $\alpha$ a great value, we wait for all the slices and so the synchronization will be efficient without margin of errors. However, a too great value for $\alpha$ will increase latency in applications. We must find a compromise in the choice of this value. We developed a prototype in order to perform this study.

\section{THE OSAGAIA COMPONENT MODEL}

The last part of our work is to define a software component model [12] in order to ensure the implementation of distributed 
multimedia applications in accordance with the specifications given by functional graphs. This model is called "Osagaia" which means "the software component" in the Basque language. The nodes of graphs will be implemented by software components. These components will be connected by means of connectors whose role is to transport media and data according to the Korrontea model.

We identify two kinds of software components: the functional ones and the nun-functional ones. Functional components are in charge of the implementation of the basic functionalities of an application. They are components of creation/capture, processing, rendering or storage. Non-functional components are in charge of the implementation of the aspects which associate non-functional properties to the applications necessary for their implementation [24]. Among these nonfunctional components, we define a component called "fusion" whose goal is to produce composed flows from a set of synchronous flows by applying the synchronization policies we defined previously. In fact, this component allows defining synchronization links introduced by functional graphs. A component which realizes the opposite function is also defined, it is called "separation". It is used to break composed flows into primitive ones. Other non-functional components are used but not described here.

The functional components need to be executed in a container whose role is to provide non-functional implementation for components. This container is called the elementary processor. Its role is to perform interactions between functional components and their outside. It is divided into two main parts: the exchange unit (composed of input and output units) and the control unit. The exchange unit manages synchronous flows input/output connections of the processor. The control unit manages the life cycle of the functional component and the interactions with the runtime platform. Thus, the platform supervises all elementary processors and indirectly all functional components.

Thanks to the elementary processor, functional components can read and write slices of synchronous flows even if these slices contain several data flows. Indeed, the functional component processes some flows, the others are transferred from input unit to output unit into the processor. When processings are achieved by the component, the output unit executes synchronization policies in order to compose slices broke by input unit. This solution is the one that we propose in order to prevent the desynchronization of flows induced by processing.

Synchronous flows are transported between elementary processor by means of a connector called the conduit. Its main role is to connect components (functional and non-functional) of applications. The conduit receives slices of synchronous flows produced by components and conveys them. It is made up of two parts. The control unit implements interactions between the conduit and the platform while an exchange unit manages the input/output connections with components. The conduit is the distributed entity of our model, i.e. it can transfer synchronous flows between different sites of distributed applications. Its internal architecture is based upon the client/server model. It constitutes the solution that we propose in order to avoid the desynchronization of flows caused by network transmission.

\section{PROTOTYPE IMPLEMENTATION}

In order to validate our approach and the different models presented in this paper, we developed several prototypes.

\subsection{Synchronization Policies Simulator}

The simulator developed allows to apply the synchronization policies on synchronous flows with both temporal constraints. It permits to produce synchronous slices of flows with only time stamps because the policies use these stamps. The aim of the simulator is to test the constitution of the composed flows. For each flow defined, we can choose its properties of the flows. The results of the constitution of the slices are displayed on a graphic interface and stored in a text file. Thus, we can analyze the results after the runtime of the simulator.

In order to create synchronous flows, the simulator uses the parameters $\theta$ and $\alpha$. Others parameters are used to indicate the number of flows with both hard and soft temporal constraint.

Each flow is created with three properties. The first one is an integer which represents the total number of data flows. The second one gives the intra-flow synchronization relation between two successive slices. For the flows with soft temporal constraint, this is the maximal time between two slices. Indeed, for these flows this time may vary. Finally, with the last property, it is possible to introduce a maximal delay for the arrival of slices at the input of the simulator. Thus, we introduce delay.

The simulator is composed of a set of windows described on Figure 5. The main window gives the constitution of the created slices of the resulting composed flow. Each slice indicates, for each flow, the number of slices used. These slices are indicated by the mean of their time stamps. The time $\mathrm{T}_{\mathrm{MAX}}$ used to constitute a slice is indicated for each resulting slice except when the soft policy is applied. The flows indicated by a capital letter are with hard temporal constraint and the flows indicated with a small letter are with soft temporal constraint. For a given slice, on flows with soft temporal constraint we can obtain the following description: $\{\mathrm{f} 0=\}$. This means that slices are available for this flow but for the moment they do not correspond to the criteria used by the policy. This kind of slices is available too early.

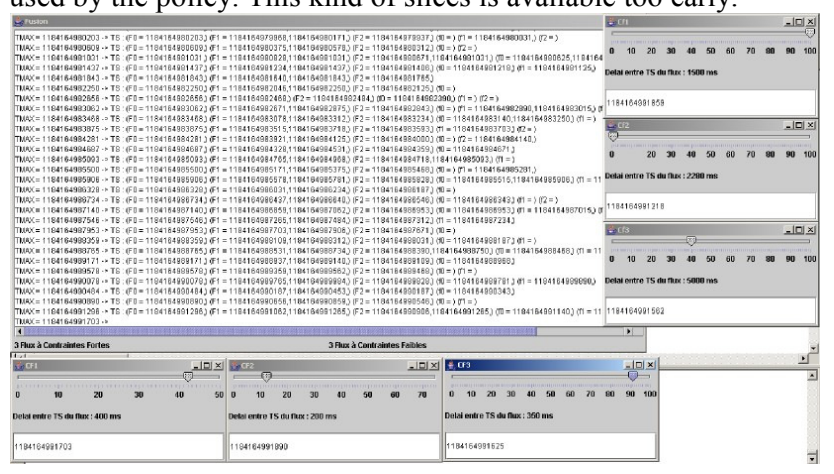

Figure 5. Synchronization Policies Simulator

This simulator can be used by the interested readers in order to test the synchronization policies of our model. This simulator can be downloaded at the following URL: http://www.iutbayonne.univ-pau.fr/ roose/V2/korronteaSimulator

\subsection{Application prototype}

We develop a prototype in order to test dynamic adaptation of an application and the synchronization policies. This prototype is distributed and is divided into two parts. The first one implements two video capture components, one from a WebCam and the other from a file. Each of these two components produces two same video flows that are put in a composed flow in order to 
keep their synchronization relations. This composed flow is transmitted to the other part of the application by the mean of a distributed conduit. This part is composed of one ore several components. This composition depends on the configurations that we want to implement. We can find a displaying component which allows to display the two video flows. We can add one ore more processing components applied on one of the two flows. These components can be added or removed dynamically. This prototype provides six processing components: an image size reduction, a negative transformation, a black and white transformation, an edge detection, a blured video and a skin color detection. Each component is executed in an elementary processor.

A graphic interface allows to simulate the runtime platform by adding or removing and starting and stopping the processing components. It permits equally to choose the source of the video flows.

The creation component produces two video flows with hard temporal constraint. Thus, the hard synchronization policy is applied. The composed flow is transmitted to the second part of the application. Then, it is transmitted to the displaying component after one or several processing components chosen by the user. The processing components receive the composed flow and apply their processing only on the second flow. The first one allows to know if the inter-flow synchronization relations between the two flows are kept. Moreover, the prototype allows to test the dynamic adaptation of the application.

The Figure 6 shows an example of the runtime of this prototype. In this case, we add an edge detection component and a component which applies a blur on the video. We can see on the video that they are synchronous despite the processing applied on the second flows.

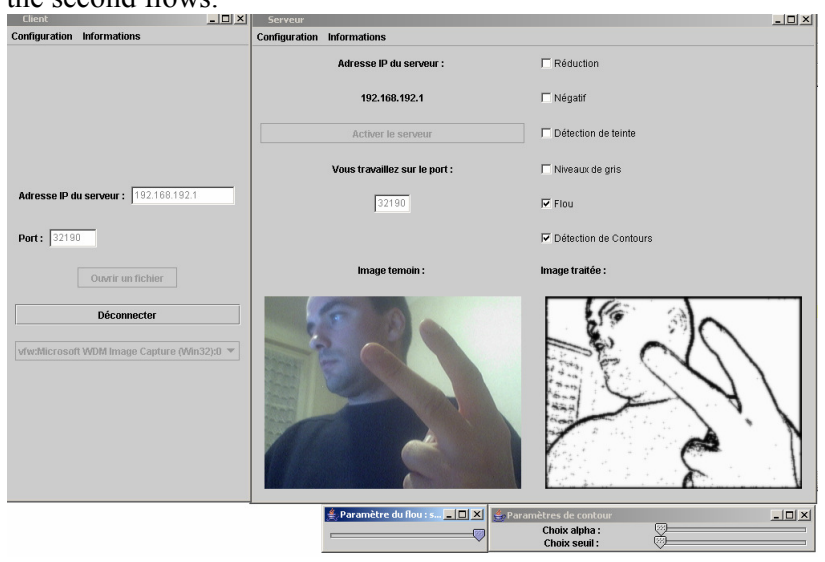

Figure 6. Edge Detection and Blur

\section{RELATED WORKS}

The development of pervasive and ubiquitous computing imposes stringent requirements that the deployment of multimedia applications must consider in order to dispose of efficient implementations. These requirements impose to adapt both applications and data. For instance, many works deal with data adaptation according to the characteristics of the runtime context. These adaptations are performed by increasing or decreasing data intrinsic quality [26]. The components paradigm allows this kind of adaptation by adding components in critical locations of a distributed application. This solution is used by our platform in order to adapt data to provided QoS.

Data must be structured in order to be handled by the entities that compose an application. Some works use coding formats like the MPEG one [27]. This kind of solution allows to consider data properties but is open to criticism for many reasons. Data integration is an important criterion in multimedia applications [5]. An MPEG solution is based on the multiplexing of several media or data. Thus, this kind of solution goes against the data integration because we loose the possibility to process single data except with the implementation of complex architectures. Indeed, this solution is not efficient due to the considerable times of compression and decompression processes. Moreover, the multiplexing of several data implies to consider a global QoS for the data. The adaptation of each media by increasing or decreasing their intrinsic quality [26] is not possible. Other disadvantages of solutions oriented multiplexing are given in [28]. Consequently, we prefer to define our own data model suitable for our works.

The properties of this data must be ensured in such applications. We identified two sources of desynchronization. The first one is due to the fact that some data synchronized with others must be processed. Processing introduces temporal delays on media and so desynchronizes the processed media in comparison with the others. The second one can occur when synchronous data are transmitted through network. Some services used to transport data on a network introduce an increase of the network load but also packet loss, delay and jitter [13]. These problems are wellknown and harmful to media synchronization [14]. Some works provide models to ensure synchronous presentation of media whose characteristics are known a priori [5]. However, it is hard to know a priori all the characteristics of data that can be used in multimedia applications. Other works provide models to allow synchronous transmission of media through networks [29]. The RTP protocol [30] gives this possibility. These works do not consider the first problem of desynchronization. We think that synchronization models are efficient only if they are extended to the whole application in order to avoid these two sources of desynchronization. With this aim, we use a data model which allows to keep synchronization relations in real-time from the source to the sink of an application.

\section{CONCLUSION}

In this paper, we presented both data and synchronization models suitable for distributed multimedia applications. From specifications given by the runtime platform, we have specified the Korrontea model used to transport data and media into applications in either local or distributed ways. We propose to use a data flow structure in order to model this data. The key idea of this research consists in exploiting the temporal dimension of the data flows that can be handled in such applications. A data flow can be built with hard or soft temporal constraint. The temporal constraint is linked to the type of the data transported into the flow. Synchronization policies that we used are based on these constraints. The Korrontea model is then used in the Osagaia component model in order to develop multimedia applications with these specifications.

The robustness of the proposed technique has been proved by the synchronization policies simulator. Another prototype allow to see that synchronization is maintained in real use case and this in spite of processings and network transfers. 


\section{REFERENCES}

[1] A. Lins et al. A Tool to Generate Multimedia Monitoring Applications for Wireless Sensor Networks. In Proceedings of the $6^{\text {th }}$ IFIP/IEEE International Conference on Management of Multimedia Networks and Services (Belfast, Northern Ireland, September 7-10 2003).

[2] T. Totozafiny et al. Motion Reference Image JPEG 2000: Road Surveillance Application with Wireless Device. In Proceedings of the Visual Communications and Image Processing (Beijing, China, July 12-15 2005).

[3] W. Zhu, N. D. Georganas. JQoS: Design and Implementation of a QoS-based Internet Videoconferencing System using the Java Media Framework (JMF). In Proceedings of the IEEE Canadian Conference on Electrical and Computer Engineering (Ontario, Canada, May 13-16 2001).

[4] A. Hafid, G. v. Bochmann, R. Dssouli. Distributed Multimedia Applications and Quality of Service: A Review. Elec. Jour. on Networks and Distributed Processing, 6 (1998), 1-50.

[5] G. Blakowski, R. Steinmetz. A Media Synchronization Survey: Reference Model, Specification, and Case Studies. IEEE Jour. on Selected Areas in Comm., 14, 1 (January 1996), 5-35.

[6] ISO/IEC/JTC1/SC29/WG12. Coding of audio, picture, multimedia and hypermedia information. MHEG Working Group S.5, June 1999.

[7] G. Ghinea, J. Thomas. QoS impact on user perception and understanding of multimedia video clips. In Proceedings of the $6^{\text {th }}$ ACM International Conference on Multimedia (Bristol, England, September 12-16 1998).

[8] R. Steinmetz. Human Perception of Jitter and Media Synchronization. IEEE Jour. on Selected Areas in Comm., 14, 1 (January 1996), 61-72.

[9] P. Eles, A. Doboli, P. Pop, Z. Peng. Scheduling with Bus Access Optimization for Distributed Embedded Systems. IEEE Trans. On Very Large Scale Integ. Sys., 8, 5 (October 2000), 472-491.

[10] S. Laplace. Conception d'Architectures Logicielles pour Intégrer la Qualité de Service dans les Applications Multimédias Réparties. Ph.D. Thesis, University of Pau, Bayonne, France, 2006.

[11] M. Dalmau, P. Roose, F. Luthon. A Distributed Architecture for Cooperative and Adaptive Multimedia Applications. In Proceedings of the $26^{\text {th }}$ IEEE Annual International Computer Software and Applications Conference (Oxford, England, August 26-29 2002.

[12] E. Bouix, M. Dalmau, P. Roose, F. Luthon. A Component Model for transmission and processing of Synchronized Multimedia Data Flows. In Proceedings of the $1^{\text {st }}$ IEEE International Conference on Distributed Frameworks for Multimedia Applications (Besançon, France, February 6-9 2005).

[13] H. E. Gebaly. Reactive Mechanisms for Recovering Audio Performance in Multimedia Conferencing Over Packet Switched Networks. Intel Tech. Jour., 3, 3 (August 1999).
[14] N. Bouillot. Métaphore de l'orchestre virtuel - Etude des contraintes systèmes et réseaux, puis prototypage. Master Thesis, IRCAM-CEDRIC/CNAM, Paris, France, 2002.

[15] L. Lamport. Time, Clocks, and the Ordering of Events in a Distributed System. Comm. of the ACM, 21, 7 (July 1978), 558-565.

[16] C. Fidge. Timestamps in message-passing systems that preserve the partial ordering. Australian Comp. Science Comm., 10, 1 (February 1988), 56-66.

[17] F. Mattern. Virtual Time and Global States of Distributed Systems. In Proceedings of International Workshop on Parallel And Distributed Algorithms (Château de Bonas, France, October 3-6 1988).

[18] C. Dumontier, F. Luthon, J-P. Charras. Real-Time DSP Implementation for MRF-Based Video Motion Detection. IEEE Trans. on Image Processing, 8, 10 (October 1999), 1341-1347.

[19] OMG. UML Profile for Schedulability, Performance and Time Specification. Version 1.1, January 2005.

[20] B. Abali, C. B. Stunkel. Time synchronization on SP1 and SP2 parallel systems. In Proceedings of the $9^{\text {th }}$ International Parallel Processing Symposium (Santa Barbara, USA, April 25-28 1995).

[21] IEEE 1588. Precision clock synchronization protocol for networked measurement and control systems. IEC $61588,1^{\text {st }}$ edition, September 2004.

[22] Sun Microsystems. Java Media Framework API Guide. November 1999.

[23] OMG. Unified Modeling Language Specification. March 2003.

[24] D. Garlan, M. Shaw. An Introduction to Software Architecture. Advances in Soft. Eng. And Knowledge Eng., 2 (1993), 1-39.

[25] Sun Microsystems. Java Sound Programmer Guide. 2000.

[26] D. Hagimont, N. Layaida. Adaptation d'une application multimedia par un code mobile. Tech. et Sciences Informatiques, 21, 6 (2002), 877-898.

[27] S-C. Chen, M-L. Shyu, N. Zhao, C. Zhang. Component-Based Design and Integration of a Distributed Multimedia Management System. In Proceedings of the 2003 IEEE International Conference on Information Reuse and Integration (Las Vegas, USA, October 27-29 2003).

[28] D. L. Tennenhouse. Layered Multiplexing Considered Harmful. In Protocols for High-Speed Networks, Elsevier Science Publishers, 1990.

[29] J-P. Courtiat and al. A General-purpose Multimedia Synchronization Mechanism based on Causal Relations. IEEE Jour. on Selected Areas in Comm., 14, 1 (Jan. 1996), 185-195.

[30] H. Schulzrinne, S. Casner, R. Frederick, V. Jacobson. RTP: A Transport Protocol for Real-Time Applications. RFC 1889, 1996. 